Commentary

\title{
Family Physician Hospitalists: the Good and the Bad
}

Satya Rashi Khare ${ }^{1}, \mathrm{MScN}, \mathrm{MBA}$

MJM 2017 15(13)

Hospitalists are physicians whose primary professional focus is the general medical care of hospitalized patients without the responsibility for care post-discharge $(1,2)$. In Canada, the majority of hospitalists are family physicians (3). Given the shortage of community-based family physicians in Canada, a growing number of hospitalists poses a unique challenge to the allocation of a scarce resource as more family physicians opt to focus their practice in a hospital setting. It is my opinion that the role of family physicians in the care of hospitalized patients is indispensable, and if threatened, will not only create a gap in inpatient care, but will also lead to reduced access to appropriate care in the community.
There was a time when family physicians practiced 'cradle-to-grave' medicine following their patients through every phase of life. When hospitalization was required, family physicians admitted their own patients and managed their inpatient care. This not only ensured continuity and comprehensiveness of care, but also gave patients a sense of security that the person most knowledgeable about their situation was at the helm of care decisions (4).

In the 1990s, Canada was experiencing a severe family physician shortage, resulting in a growing number of orphaned patients; patients admitted to hospital who did not have a family physician. These patients were assigned to family physicians who were

${ }^{1}$ Department of Family Medicine, McGill University, Montréal, Canada. Corresponding Author: Satya Rashi Khare, email satya.khare@mail.mcgill.ca. 
following their own patients in the hospital, increasing their workload beyond sustainability (5). Concurrently, remuneration for hospital work was inadequate, care complexity was rising, and outpatient volumes were increasing (3). This combination of excessive work for modest income led to many family physicians resigning their hospital privileges. In response, hospitals created hospitalist groups staffed by family physicians dedicated to inpatient care - called hospitalists (6). More recently, hospitalist specialties have emerged in areas such as neurology, surgery, and obstetrics and gynecology (2).

The greatest drawback to this model of hospital care is aggravating an existing shortage of family physicians in the community. This has led the College of Family Physicians of Canada (CFPC) to actively promote family physician hospitalists to also spend time in community practice (5). However, balancing outpatient and inpatient care can be overwhelmingly demanding. For example, recent health care reform in Québec has placed pressure to see more patients in the office (in the context of Bill 20) while maximizing efficiency in the hospital (in the context of Bill 10). This could eventually lead to burnout and work-life balance issues for family physicians who practice both community-based and hospital-based work.

Further drawing family physicians away from community-based practice is the attractive compensation of hospital-based care. Although physician compensation in Canada is largely fee-forservice, hospitalists are often on a blended payment model with fee-for-service income 'topped-up' with salary support by the hospital (7). Moreover, the allure of hospital medicine includes the opportunity to practice higher acuity medicine; something that may be more attractive to some family physicians.

A more fundamental concern may relate to whether or not hospital-based medicine satisfies the core values defined in family practice, such as continuous, comprehensive, and patient-centered care that is based on a trusting patient-physician relationship (8). Although some principles are indeed met in the practice of hospital medicine, others are simply unattainable in a chaotic, time-pressured, acuteoriented environment. This begs the question of whether hospital medicine threatens what the discipline of family medicine represents.

Finally, despite the CFPC approving the creation of a hospital medicine program, in addition to many post- graduate enhanced skills training programs, there are no national certification or training guidelines for Canadian hospitalists (2). This is not surprising as hospitalist groups in Canada emerged at the level of individual hospitals so the scope of practice was not predefined and nationwide guidelines were not established (6).

Given these concerns, what would happen if family physician hospitalists cease to exist? Favorably, the shortage of family physicians in community-based practice would be somewhat alleviated as outpatient volumes could be better accommodated. Unfavorably, major gaps in inpatient care would be created. These gaps would have to be filled with specialists in the hospital which could lead to incomplete coverage given their short supply and busy outpatient clinics, thus reducing the ability to respond to rapidly changing needs of inpatients. Furthermore, time sharing between inpatient and outpatient care would inevitably lead to a reduction in outpatient volumes which would in turn cause increased waiting times for patients referred by family physicians in the community. This scenario is more relevant to urban centers where specialists provide care to hospitalized patients. In rural centers that do not have specialists, inpatient physician coverage would be highly problematic without family physician hospitalists.

Additional advantages of family physician hospitalists relate to the core values of family practice. Patients admitted to hospital, often in a very vulnerable state, could benefit greatly from the holistic and patient-centered approach that comprises one of the key principles of family medicine (4). Relatedly, family physicians are generalists and thus have a broad skill set in managing multiple ailments, and also play a fundamental role in coordinating care between multiple care providers. With a growing influx of complex patients, these tenets of family medicine may optimize inpatient care. Though more research is needed, some studies have shown shorter length-of-stays and lower cost per stay with the use of hospitalists verses specialists $(1,9)$.

In my view, the challenges for maintaining the family physician hospitalist model going forward are formidable. Adequate training requirements, definition of scope of practice, design of optimal workload to balance outpatient and inpatient care, system sustainability measures to avoid burnout, and creation of more equalized remuneration models are a few that come to mind. Furthermore, research on the effect of 
Canadian hospitalists on efficiency, quality of care, and patient satisfaction is needed.

The hospitalist movement is continuing to grow - a 2012 national physician survey showed that $49 \%$ of family medicine residents intended to practice in a hospital setting (10). Despite concerns related to family physician hospitalists - less time in community practice, potential misalignment with core discipline values, and lack of regulation - it is my belief that removing family physicians from the hospitalist role will create major gaps in inpatient care and threaten access to care in the community. In essence, inpatient care will suffer through reduced medical presence on the ward, outpatient specialist care will suffer through reduced volume as specialists share their time between clinics and wards, and primary care will suffer through increased waiting times for specialist, secondary, care. As such, family physician hospitalists should be embraced and supported through definition of the model in more certain terms and nationwide standardization.

\section{References}

1. Hock Lee K, Yang Y, Soong Yang K, Chi Ong B, Seong $\mathrm{Ng} \mathrm{H}$. Bringing generalists into the hospital: outcomes of a family medicine hospitalist model in Singapore. Journal of Hospital Medicine. 2011;6(3):115-21.

2. White HL, Stukel TA, Wodchis WP, Glazier RH. Defining hospitalist physicians using clinical practice data: a systems-level pilot study of Ontario physicians. Open Medicine 2013;7(3):e74-e84.

3. Soong C, Fan E, Howell EE, Maloney RJ, Pronovost PJ, Wilton D, et al. Characteristics of hospitalists and hospitalist programs in the United States and Canada. Journal of Clinical Outcomes Management. 2009;16(2):69-74.

4. The College of Family Physicians of Canada. Family physicians caring for hospital inpatients: a discussion paper prepared by the College of Family Physicians of Canada Mississauga, ON: Arch Fam Med 2003. p. 42.

5. Lee $\mathrm{KH}$. The hospitalist movement - a complex adaptive response to fragmentation of care in hospitals. Annals Academy of Medicine Singapore. 2008;37(2):145-50.

6. Smith SD, Sivjee K. Defining training needs, core competencies and future certification for Canadian hospitalists. Canadian Medical Association Journal. 2012;184(14):1557-8.

7. National Physician Survey. Fast facts: Hospitalist care Canadian Family Physician 2008;54:1145.

8. The Ontario College of Family Physicians. Comprehensive and continuous care in a collaborative care environment: challenges for 21 st century family medicine and hospitals Toronto, ON 2008.
9. Peterson MC. A systematic review of outcomes and quality measures in adult patients cared for by hospitalists vs nonhospitalists. Mayo Clinic Proceedings. 2009;84(3):248-54.

10. The College of Family Physicians of Canada, Canadian Medical Association, The Royal College of Physicians and Surgeons of Canada. National Physician Survey, 2012. Results for Medical Residents. Mississauga, ON 2012 [Available from: http://nationalphysiciansurvey.ca/wpcontent/uploads/2013/02/NPS2012-Resident-FullEN.pdf. 


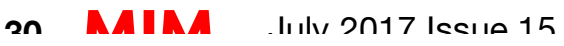

